\title{
Review Article \\ DNA Methylation and Chromatin Remodeling: The Blueprint of Cancer Epigenetics
}

\author{
Dipanjan Bhattacharjee, Smita Shenoy, and Kurady Laxminarayana Bairy \\ Department of Pharmacology, Kasturba Medical College, Manipal University, Manipal, Karnataka 576104, India \\ Correspondence should be addressed to Dipanjan Bhattacharjee; dipanjan_bhttchrj@rediffmail.com
}

Received 30 December 2015; Accepted 10 March 2016

Academic Editor: Yubin Ge

Copyright ( 2016 Dipanjan Bhattacharjee et al. This is an open access article distributed under the Creative Commons Attribution License, which permits unrestricted use, distribution, and reproduction in any medium, provided the original work is properly cited.

\begin{abstract}
Epigenetics deals with the interactions between genes and the immediate cellular environment. These interactions go a long way in shaping up each and every person's individuality. Further, reversibility of epigenetic interactions may offer a dynamic control over the expression of various critical genes. Thus, tweaking the epigenetic machinery may help cause or cure diseases, especially cancer. Therefore, cancer epigenetics, especially at a molecular level, needs to be scrutinised closely, as it could potentially serve as the future pharmaceutical goldmine against neoplastic diseases. However, in view of its rapidly enlarging scope of application, it has become difficult to keep abreast of scientific information coming out of various epigenetic studies directed against cancer. Using this review, we have attempted to shed light on two of the most important mechanisms implicated in cancer, that is, DNA (deoxyribonucleic acid) methylation and histone modifications, and their place in cancer pathogenesis. Further, we have attempted to take stock of the new epigenetic drugs that have emerged onto the market as well as those in the pipeline that offer hope in mankind's fight against cancer.
\end{abstract}

\section{Introduction}

Tumorigenesis, the process of development of cancerous cells, till recent times, was believed to be a by-product of aberrant genetic mutations alone. However, recent evidences have pointed to an important role of epigenetics in the development of cancer. The term "epigenetics," a portmanteau of "epigenesis" and "genetics," can be summed up in the following few words: "Epigenetics refers to both heritable changes in gene activity and expression (in the progeny of cells or of individuals) and also stable, long-term alterations in the transcriptional potential of a cell that are not necessarily heritable" [1]. The field of epigenetics revolves around the concept of "epigenetic landscape" at its core. In a very rudimentary form, epigenetic landscape has been designated to represent the intra- and extracellular environment and epigenetics refers to the study of the various interactions between the intracellular and extracellular environment that in turn decide the cell's fate. It has been hypothesised that the "epigenomic landscape," governing the cellular expression, becomes extensively warped in cancerous cells [2]. This distortion is due to the deviations from the regular DNA methylation and histone modification patterns seen in normal cells. These alterations in the epigenetic regulatory machinery can bring about dysregulation in genetic expression patterns either by the activation of the oncogenes [3] or by the silencing of tumor suppressor genes. Thus, epigenetic dysregulation can lead to development and perpetuation of cancerous states. However, epigenetic regulatory machinery, being reversible and dynamic in nature, is shorn of the rigidity associated with genetics and possesses the potential to either cause or cure diseases, especially cancer. Hence, epigenetics, especially at a molecular level, needs to be examined closely, as it could serve as the future pharmaceutical goldmine. In light of this, it is our endeavour here to shed light on the implications of the two most prominent epigenetic mechanisms, that is, DNA methylation and histone modification, in the pathophysiology of cancer. Further, we attempt to take stock of the new epigenetic drugs that have emerged onto the market as well as those in the pipeline that offer hope in mankind's fight against cancer. 


\section{Cancer and DNA Methylation}

DNA methylation primarily involves addition of methyl groups to the $5^{\prime}$ carbon at cytosine residues preceding guanine nucleotides, linked together by phosphate bonds $(\mathrm{CpG})$ utilizing a methyl donor like S-adenosylmethionine. The $\mathrm{CpG}$ rich foci are asymmetrically arranged throughout the genome, clustered primarily in short $\mathrm{CpG}$ rich DNA sequences termed as "CpG islands" and regions of large repetitive sequences like centromeric repeats, retrotransposon elements, and so forth $[4,5]$. DNA methylation, brought about by a class of enzymes termed as DNA methyl transferases (DNMT), target these CpG islands. There exist four DNMTs, that is, DNMT 1, 2, and 3a and 3b [6] of which DNMT 1 and DNMT 3 have been observed to play a pivotal role in DNA methylation.

DNA methylation can deter the process of transcription by inhibiting the binding of the transcriptional factors with the target sites as seen in case of $c-m y c$ and many other genes [7]. On the other hand, the methylated cytosine residues act as the site for docking of various methylated DNA binding proteins (MBD1, MBD2, MBD3, and Mecp2) that are recognized by various histone modifying enzymes like histone deacetylases (HDACs), which in turn can bring about gene repression [8-10].

A normal cell is characterized by genome wide methylation with the exception of CpG (cytosine-phosphateguanine) islands, which are unmethylated [11]. However, by virtue of various triggers in cancerous cells, whose origins are yet to be understood properly, certain events are set in motion which lead to the hypomethylation of entire genome with the exception of $\mathrm{CpG}$ island promoters, which undergo hypermethylation [12].

\subsection{Role of Hypomethylation in Cancer. Extensive DNA hypo-} methylation is essential for tumorigenesis as its occurrence at repetitive elements can produce an increase in genomic instability by advocating chromosomal rearrangements $[12$, 13]. Further, retrotransposons' hypomethylation leads to their activation. This in turn can lead to the translocation of retrotransposons to other genomic regions, which in turn could propagate the genomic instability [14]. Some of the best examples to exemplify the role of DNA hypomethylation in development of cancer have been illustrated here. DNA hypomethylation has been implicated in the activation of the growth promoting genes such as related-RAS (R-Ras) and mammary serine protease inhibitor (MAPSIN) for gastric carcinoma, S100 in case of colonic cancer, melanoma-associated antigen (MAGE) in melanoma [15], and loss of imprinting, especially of insulin like growth factor 2 (IGF-2) as seen in Wilms' tumor [16] and colorectal cancer [17].

2.2. Role of Hypermethylation in Cancer. Contrastingly, $\mathrm{CpG}$ island hypermethylation can induce tumorigenesis by shutting down the expression of tumor suppressor genes. This can be achieved by direct action over tumor suppressor genes as well as indirectly by silencing of the concerned tumor suppressor genes' transcription factors and preventing the expression of DNA repair genes. Hypermethylation of $\mathrm{Rb}$ promoter gene (retinoblastoma associated tumor suppressor gene) was among the earliest instances to have been unearthed, where hypermethylation of the $\mathrm{CpG}$ promoter island site led to the silencing of the tumor suppressor gene and subsequently promotion of retinoblastoma malignancy [18]. A few other such tumor suppressor genes that undergo silencing due to hypermethylation include p16 and BRCA1 [19]. These genes are crucial to processes like cellular adhesion, apoptosis, and angiogenesis that are involved in the development and progression of cancer. On the other hand, hypermethylation of CpG promoter regions induced silencing of transcription factors as seen with RUNX3, GATA-4, and GATA-5 in esophageal, colorectal, and gastric cancers, respectively, leads to downstream target inactivation of the tumor suppressor genes, which in turn can lead to propagation of cancer cells [20,21]. Further, DNA repair genes like MLH1 and BRCA1 on being silenced allow the accrual of many other genetic lesions, which in turn accelerates the progression of cancer.

In the light of growing evidence that progressively bolstered the claim that DNA hypermethylation plays a critical role in tumorigenesis, questions then arose as to how can selective targeting of genes by the DNA methylation machinery be carried out? One line of thinking is that $\mathrm{CpG}$ island specific methylation may be guided by a nucleotide sequence specific mechanism that in turn directs the DNMTs to their respective genes based upon their association with the oncogenic transcription factors. PML-RAR fusion protein guided abnormal hypermethylation and specific target promoter genes' silencing as seen in acute promyelocytic leukemia are a classic example to illustrate this [22]. Further, as has been observed in various types of cancer, large stretches of DNA tend to undergo methylation. This may lead to hypermethylation of the $\mathrm{CpG}$ islands by virtue of them being located within genomic regions that may have undergone large scale epigenetic reprogramming [23]. Apart from these two possible mechanisms, it has also been hypothesised that histone marks may also play a significant role in $\mathrm{CpG}$ island specific de novo DNA hypermethylation. Further light has been shed over this mechanism in the subsequent sections.

\subsection{TET Proteins: The Master Key of DNA Methylation.} Despite hypomethylation and hypermethylation producing diametrically opposite results in cancerous tissues, both these mechanisms tend to coexist in the same tumor, though afflicting different regions within the genome. Both these mechanisms can interact in many manners and at various levels to produce multiple subphenotypes of cancer. Further, the reversibility of DNA methylation epigenetic process adds to the complexity of the cancerous tissue's epigenome as it renders the epigenetic regulatory processes open to modification by various cellular environment changes. This again goes against the belief that DNA methylation could be a stable form of chromatin modification. High resolution pangenome DNA methylation mapping in pluripotent and differentiated cells has further underlined the flexible nature of DNA methylation. This could be attributed to the presence of an enzymatic system that may be responsible for either completely abolishing or maybe altering this epigenetic modification [24]. This hypothesis, attempting to explain the dynamic 
TABLE 1: DNMT inhibitors in cancer.

\begin{tabular}{|c|c|c|}
\hline Drug & Therapeutic use & Developmental stage \\
\hline \multicolumn{3}{|l|}{ Nucleoside analogue inhibitors } \\
\hline \multirow{3}{*}{ (1) 5-azacytidine } & Myelodysplastic syndrome & $\begin{array}{l}\text { Approved [United States-Food and Drug } \\
\text { Administration (US-FDA)] }\end{array}$ \\
\hline & Acute myeloid leukemia & Phase 2 \\
\hline & Solid tumors & Phase 2 \\
\hline \multirow{2}{*}{ (2) Decitabine } & Myelodysplastic syndrome & Approved (US-FDA) \\
\hline & Acute myeloid leukemia & Approved [European Commission (EC)] \\
\hline (3) Zebularine & $\begin{array}{l}\text { Solid tumors like breast, urinary bladder, } \\
\text { hepatocellular cancer }\end{array}$ & Preclinical \\
\hline \multirow{3}{*}{ (4) SGI-110 } & Myelodysplastic syndrome & Phase 1 \\
\hline & Acute myeloid leukemia & Phase 1 \\
\hline & Solid tumors like bladder cancer & Preclinical \\
\hline \multicolumn{3}{|c|}{ Nonnucleoside analogue inhibitors } \\
\hline \multicolumn{3}{|c|}{$\begin{array}{l}\text { (1) Procainamide } \\
\text { (2) Procaine } \\
\text { (3) Epigallocatechin-3-gallate }\end{array}$} \\
\hline (4) SGI-1027 & Leukemia & Preclinical \\
\hline \multirow{3}{*}{ (5) Hydralazine } & Breast cancer & Phase 2 \\
\hline & Ovarian cancer & Phase 3 \\
\hline & Cervical cancer & Phase 3 \\
\hline
\end{tabular}

nature of DNA methylation, received a shot in the arm with the discovery of ten-eleven translocation (TET1-3) group of proteins. TET group of proteins is etymologically associated with a recurrent chromosomal translocation $\mathrm{t}(10 ; 11)(\mathrm{q} 22 ; \mathrm{q} 23)$ that apposes mixed-lineage leukemia or myeloid-lymphoid leukemia (MLL) gene with TET1 protein in a few acute myelocytic leukemia (AML) patients. This family of proteins functions as mammalian DNA hydroxylase that converts 5-methyl cytosine $(5 \mathrm{mC})$ to 5-hydroxymethylcytosine $(5 \mathrm{hmC})$, which, on further oxidation, ends up yielding various oxidation products, like 5 -formylcytosine (5 fC) and 5-carboxylcytosine $(5 \mathrm{caC})$. Though further studies are needed to fully establish the biological role of these oxidation derivatives, it is believed that these derivatives could be the critical intermediates in DNA methylation, be it active or passive. Further, they could also play an important role in either preventing or even enhancing the attachment of methyl $\mathrm{CpG}$ binding domain (MBD) proteins and could also regulate the recruitment of chromatin regulators. Additionally, the genome wide distribution of $5 \mathrm{hmC}$ gives rise to the notion that $5 \mathrm{hmC}$ and TET proteins in all probability may influence both transcriptional activation and silencing [25].

2.4. DNA Methylation and Its Therapeutic Applications. In light of the dynamic and far reaching effects of aberrant DNA methylation, it is not surprising to note that hypomethylating agents (Table 1) were among the first epigenetic therapies to obtain the United States Food and Drug Administration (USFDA) approval.

The hypomethylating agents have created a niche for themselves among hematological malignancies, most notably against myelodysplastic syndrome (MDS) [26-30]. However, the application of these hypomethylating agents/DNMT inhibitors (DNMTi) in solid malignancies has met with a snag as the results have not been very encouraging [31, 32]. This could have resulted from the much more complex nature of solid tumors as compared to hematological neoplasms [33]. Another possibility could be due to the slow rate of replication dependent incorporation of the DNMTi agents in the solid tumor cells. Besides, this group of drugs gets inactivated by cytidine deaminase enzyme. Further, the DNMTi agents are effective against solid tumors, at much higher doses than the low doses at which they are effective against hematological malignancies, thus giving rise to the problem of severe toxicity. However, results of a phase 2 trial [34] clearly attest to the fact that low-dose azacytidine can be utilized for chemosensitisation of solid tumors to conventional chemotherapy, thus providing a new direction to the application of DNMTi against solid tumors and paving the way for future clinical studies in this direction.

Another unique strategy to achieve gene demethylation is by using small nonnucleoside DNMT inhibitor molecules (Table 1) that partially competitively inhibit DNMT 1 and decrease the affinity of DNMT for its substrates, thus finally favouring the dissociation of DNMT 1 from hemimethylated DNA. Besides monotherapy, the DNMT inhibitors, for instance, the azanucleosides, have shown excellent efficacy in combination with standard nucleoside analogues like 5fluorouracil as the DNMT inhibitors can reignite the dormant or silenced proapoptotic genes $[35,36]$. Additionally, it has also emerged that HDAC inhibitors and DNMTi when used synergistically may yield superior results, thus potentially 
opening up another manner of utilization of these agents [3739].

\section{Histone Modification and Cancer}

Despite DNA methylation being discovered earlier, however, in the recent years, it is the histone modification that has slowly risen to prominence in research in cancer. The remarkable diversity afforded by the versatile histone modifications is slowly being unravelled. The ability of multiple histone modifications to coexist, not all of them being activating in nature as evidenced by the discovery of "bivalent domains," that is, the coexistence of activating and repressive marks at the promoter sites of developmentally critical genes, provides a dynamic and complex epigenetic landscape, where genetic expression can be modulated at various levels and in different manners. Let us then try to elaborate on the role played by different types of histone modifications in cancer.

3.1. Histone Acetylation: An Untapped Gravy Train. Histone acetylation, a very dynamic histone modifying interaction, is carried out by histone lysine acetyltransferases (KATs). There exist 2 different types of KATs, that is, types A and B that carry out histone acetylation of nuclear and cytoplasmic histones, respectively. KATs are among the pioneering enzymes to have been implicated in cancer [40]. Numerous instances implicating KATs in various recurrent chromosomal translocations (e.g., MLL-CBP [41], MOZ-TIF2 [42]) and coding mutations (e.g., p300/CBP $[43,44]$ ) in a wide variety of solid and hematological malignancies have been observed. The research into the role of KAT in the causation of malignancies is still in its nascent stages. However, information regarding the consequences of certain translocations has emerged now. It is believed that the MOZ-TIF2 translocation may lead to a return of aggressive leukemia as seen in animal models. Further, it can confer pluripotency and may lead to the reinitiation of self-replicating ability, if introduced into committed hematopoietic progenitor cells. The oncogenic potential of this translocation has a lot to do with the intrinsic and acquired KAT activity [42, 45]. Despite it being apparent that histone acetylation is disturbed in cancers [46, 47], it is extremely interesting to note that several nonhistone proteins also undergo dynamic acetylation, among which oncogenes and tumor suppressor genes like MYC, p53, and PTEN are also included [48]. This has posed a big conundrum towards obtaining a lucid understanding of the specific molecular mechanisms via which KATs contribute to human malignancies. Further, the endeavour aimed at development of specific inhibitors of KAT families has often been fraught with frustration over the issue of specificity of action [49]. But the recent successes so obtained with various derivatives of natural KAT 1 containing compounds in the form of garcinol and curcumin, to name a few, has raised the hopes of the scientific community that specific KAT inhibitors could possibly be synthesised in the near future [49].

3.2. Histone Deacetylation: Lying in Wait for Its Second Coming. Histone deacetylation, induced by HDACs, is another epigenetic target that is being explored aggressively in the field of cancer. HDACs carry out the reversal of acetylation at lysine residues. As noted earlier in this review, there exist 4 different classes of HDACs. Classes I, II, and IV HDACs act in a similar manner that requires the presence of a zinc metal ion at the catalytic site, but not a cofactor. However, class III HDACs, especially Sirtuins $1-7$, require NAD $^{+}$(nicotinamide adenine dinucleotide) as a cofactor for their activity. It has been observed that the level of HDACs varies significantly in various types of cancers, especially in case of hematological malignancies. The various chimeric fusion proteins like PMLRAR $\alpha$ and AML1-ETO seen in leukemia have been observed to attract various HDACs with an aim of inducing abnormal gene silencing. This in turn contributes to perpetuation of leukemia [50]. Thus, it was hypothesised that inhibitors of the HDACs could in turn lead to reversal of aberrant gene silencing, which could cascade out to induction of growth arrest, differentiation, and apoptosis of the malignant cells $[50,51]$. This paved the way for various studies that were carried out with the sole aim of identifying and examining the efficacy of various agents that could reverse the action of HDACs in various types of cancers [50, 51]. Earliest instance of HDAC inhibition, although weak, was recognized with sodium butyrate which included sodium valproate and phenyl butyrate [52-56]. Since then, a range of more potent and structurally diverse HDACi have been obtained naturally or synthetically produced.

Vorinostat, a pan HDACi (inhibitor of classes I and II HDAC enzymes), was granted US-FDA approval on 6 October, 2006, as an oral agent against cutaneous T-cell lymphoma, which can be progressive and persistent and relapsed after 2 cycles of systemic chemotherapy on the basis of phase 1 [57] and phase 2 clinical trials $[58,59]$. It acts by binding to the active site of histone deacetylases and chelating the zinc ions found on the active site, thus preventing the deacetylation from occurring, which via cascade of events promotes cellular differentiation. Vorinostat has exhibited activity in various other hematological [60-64] and solid tumors [65], thus making it a promising drug in the years to come.

Another HDACi agent, romidepsin (depsipeptide, FR901228, FK228, and NSC 630176), a natural product obtained from Chromobacterium violaceum, received USFDA approval as a second-line agent for cutaneous T-cell lymphoma on 5 November, 2009. Romidepsin, a prodrug upon entering the cell, is converted into an active compound that preferentially interacts with the zinc at the active site of the HDAC class I enzymes. But it does exert certain amount of inhibitory activity over class II HDAC enzymes [66]. Results from multiple phase 1 trials $[67,68]$ and subsequently two phase $2[69,70]$ trials with romidepsin showed good response rates. These trials revealed romidepsin to possess a very long duration of action, extending beyond 3 years in some patients, even after discontinuation of the drug, and belied the fear of possessing pronounced cardiac side-effects [71].

In the same class of drugs, MGCD0103, an isotype specific aminophenylbenzamide that inhibits HDAC I and IV enzymes, was being investigated in clinical trials for its utility in various hematological tumors $[72,73]$ and lymphomas [74]. However, recent reports of pericarditis and pericardial effusion have thrown a spanner in the development process of 
TABLE 2: HDAC inhibitors in developmental phases.

\begin{tabular}{llc}
\hline Drug & Therapeutic use & Developmental stage \\
\hline (1) Panobinostat & Cutaneous T-cell lymphoma & Phase 3 \\
\hline (2) Valproic acid & Cervical cancer & Phase 3 \\
& Ovarian cancer & Phase 3 \\
\hline (3) Belinostat & Ovarian cancer & Phase 2 \\
\hline \multirow{2}{*}{ (4) Mocetinostat } & Follicular lymphoma 2 & Phase 2 \\
& Hodgkin's lymphoma & Phase 2 \\
\hline (5) Abexinostat & Acute myeloid leukemia & Phase 2 \\
\hline & Sarcoma and lymphoma & Phase 2 \\
(6) Entinostat & Hodgkin's lymphoma & Phase 2 \\
& Breast cancer & Phase 2 \\
\hline (7) SB939 & Metastatic lung cancer & Phase 2 \\
\hline (8) Resminostat & Prostate cancer & Phase 2 \\
\hline (9) Givinostat & Hodgkin's lymphoma & Phase 2 \\
\hline (10) Kevetrin & Hepatocellular carcinoma & Phase 2 \\
\hline (11) ACY-1215 & Leukemia and lymphoma & Preclinical \\
\hline
\end{tabular}

this drug. Panobinostat (LBH-589) is being developed, with the primary focus being against hematological malignancies [75-77], refractory or relapsed T-cell lymphomas [78, 79], Hodgkin's lymphomas [80], and Waldenstrom macroglobulinemia [81]. Recently, the results of PANORAMA 1 and 2 trials [82] exhibited that panobinostat if added to bortezomib and dexamethasone could significantly extend the progression-free survival period in refractory or relapsed multiple myeloma when compared to bortezomib plus dexamethasone regimen alone [83]. This in turn led to the US-FDA granting panobinostat "PRIORITY REVIEW" designation as a new drug for multiple myeloma in May, 2014. Belinostat (PXD 101), a novel inhibitor of enzymatic activity of class 1 and class 2, has been approved by the US-FDA on 03 July, 2014, for relapsed or refractory peripheral T-cell lymphoma. However, belinostat has not shown similar efficacy against solid tumors $[84,85]$. Other HDACi in various stages of development have been listed in Table 2 .

Among other molecules that exert their effects via modulation of histone modifications, class III HDACs, known as Sirtuins or the silent information regulator 2 (sir2) family of proteins, have captivated the interest of scientific community in the last decade. This can be attributed to their critical role in several biological processes, which in turn has sparked off multiple studies aimed at exploring their activity against diseases across the spectrum, with special emphasis on tumors. The most potent sirtuin activator, and the first to be characterized, resveratrol (3,5, $4^{\prime}$-trihydroxy-trans-stilbene), is a nonflavonoid polyphenol, first isolated in 1940 [86] as an ingredient from the roots of white hellebore (Veratrum grandiflorum O. Loes). Based upon its ability to influence various cellsignaling molecules, resveratrol is being explored for its chemosensitising property [87-91] and ability to prevent cancer [92]. However, similar to KATs, HDACs too suffer from issues related to nonspecificity of their activity. Besides interacting with histone proteins, HDACs further interact with nonhistone substrates like nonchimeric oncogenes BCL6 [93]. Thus, presently, despite the large number of molecules being investigated as HDAC inhibitors, the offtarget effects of these agents still manage to confound the researchers and pose the single biggest hurdle towards their emergence as an effective therapeutic option against cancer.

\subsection{BET (Bromodomain and Extraterminal Domain Family)} Inhibitors: The New Kid on the Block. In addition to histone acetylation and deacetylation, another avenue for exploration in the form of histone acetylation readers has slowly emerged that has been shown to exert influence over genetic expression. These readers comprise a binding motif termed as bromodomain that is very well conserved over generations [94]. Of the many groups of proteins belonging to the family of histone acetylation readers, recent studies have illustrated that the BET family containing the bromodomain proteins (BRD2, BRD3, BRD4, and BRDt) can be successfully inhibited $[95,96]$. The proteins belonging to the BET family possess tandem amino-terminal bromodomains that are highly conserved sequentially. Targeting the BET family of proteins assumes significance as they are fundamental to transcriptional elongation and cell-cycle progression [96]. BET inhibitors have been found to be very effective against nuclear protein in testis- (NUT-) midline carcinoma [96] and in a wide spectrum of hematological malignancies [97-100]. It has been hypothesised that the antineoplastic activity of BET inhibitors could be attributed to myelocytomatosis (MYC) transcription downregulation. MYC is a master regulatory gene for cellular proliferation and survival and is very commonly dysregulated in neoplastic conditions [101]. However, the results of a few studies have produced evidences that 
suggest that the effects of BET inhibition may not be solely due to MYC inhibition [97-99]. Amidst all the controversy surrounding BET inhibitors, it has however emerged that BET inhibitors can specifically modulate a small set of genes, primarily by inhibiting transcription elongation. Further studies are needed to decipher the other mechanisms by which BET inhibition can occur and the associated efficacy of each mechanism that can be translated into potential anticancer therapeutic options.

\subsection{Histone Methylation and Demethylation: The Rising Star} of Cancer Epigenetics. Histone methylation can occur at arginine, histidine, or lysine residues on the histone side chains. However, we shall limit our discussion to methylation of lysine residues only as they characterize histone methylation best. The enzyme implicated here, that is, histone lysine methyltransferases (KMT), unlike the KATs possesses the ability to target specific lysine residues, thus enabling the scientists to leapfrog the issue of off-target effects that has often proven to be a big hindrance in the development of epigenetic agents. Various cancers have been studied in relation to a large number of KMTs that include MMSET, EZH2, and MLL family members. However, in recent years, among the KMTs, it is the paradoxical role of EZH2 in human malignancies that has captured the imagination of the epigeneticists [102]. Overexpression of EZH2 which is responsible for $\mathrm{H} 3 \mathrm{~K} 27$ methylation, in prostate and breast cancer, was found to be associated with poor prognosis [103, 104], suggestive of EZH2 being an oncogene. However, a few studies also showed that coding mutations in EZH2 were present in various lymphoid and myeloid neoplasms, which implied that EZH2 could potentially have a tumor suppressive role [105-108]. The exactness of the mechanisms by which $\mathrm{EZH} 2$ impacts cancer needs further investigation. However, in recent times, a better understanding of the role of KMTs in various types of cancers has accelerated the search for specific KMT inhibitors, especially in the light of the promise exhibited by small molecule inhibitors in MLL leukemia [109].

Alternatively, the discovery of two different classes of histone lysine demethylases (LSD) forced the scientists to reconsider the earlier held notion that histone lysine methylation was a stable epigenetic modification. The first class of lysine histone demethylases, that is, LSD1/KDM1A, carries out amine oxidation leading to demethylation of lysine in presence of flavin adenine dinucleotide (FAD) as a cofactor. However, its activity is limited to mono- and dimethyl lysine as it requires a protonated nitrogen atom for its activity. On the other hand, the second class of histone lysine demethylases, that is, Jumonji demethylases, with a well-conserved JmjC domain, functions through an oxidative and radical attack mechanism. As the Jumonji class of enzymes does not require a free protonated nitrogen atom to initiate its activity, the spectrum of substrates on which it can exert its activity also includes trimethyllysine. Further, these enzymes' activity is guided by the multisubunit complexes within which they reside that in turn are responsible for the specificity of their activities. For instance, LSD1 functions as a repressor of transcription when it is associated with the corepressor for RE1silencing transcription factor (Co-REST) complex. However, it functions as an activator, when it is associated with androgen receptor [110]. Research into the role of histone methylation and demethylation in cancer is still in its infancy. However, the emergence of data from various preclinical studies indicating their therapeutic potential in acute myeloid leukemia (AML) will further spur on the development of histone demethylases inhibitors, which are already at various stages of development [111, 112].

3.5. Histone Methylation Readers and Histone Phosphorylation: The Path Less Trodden. Analogous to DNA methylation readers, histone lysine methylation too is recognized and analysed by various proteins that possess special motifs. These specialised domains can be broadly classified as the Royal Family including Tudor domains, Chromo domains, malignant brain tumor domains, and PHD fingers [113]. Recent studies have come up with evidences to implicate a few of the lysine methylation readers in various types of cancers. For instance, the expression of a chromodomain protein HP1 has been observed to be altered [114]. Another example in the form of coding mutations among ING family members that specifically recognizes $\mathrm{H} 3 \mathrm{~K} 4 \mathrm{me} 3$, being identified in melanomas and breast cancer, points out to the potential of histone methylation readers being developed as antineoplastic agents [115]. Further, a recent study examined AML induced by the fusion of NUP98 and PHD finger containing a segment of JARID1A or PHF23. It was observed that mutations that counter PHD finger's ability to bind to H3K4me3 could indeed suppress the development of this particular variant of AML [116]. Thus, despite the paucity of research in this section of epigenetics, the above instances do exemplify the potential that histone lysine methylation readers possess as antineoplastic agents.

Besides histone methylation, another highly dynamic posttranslational histone modification that is now attracting the attention of epigeneticists is histone phosphorylation. Histone phosphorylation is essential to various cellular phenomena, for instance, mitosis and apoptosis. It is regulated by kinases and phosphatases that carry out phosphorylation and dephosphorylation, respectively. Though very little is known about the role of phosphatases outside the realms of DNA repair and mitosis regulation, kinases on the other hand have been studied at great length. Till recently, kinases' role was thought to be limited to signal transduction in the cytoplasm. However, now, certain kinases are believed to be responsible for histone phosphorylation [117-119], most prominent of which is JAK2 (Janus Kinase 2). JAK2 has been observed to be overexpressed in various hematological malignancies. It has been shown to phosphorylate H3Y41, thus preventing the binding of chromatin repressor HP1 $\alpha$ and leading to the activation of various hematological oncogenes. Further, the research into JAK enzymes broadened out to include Hodgkin's lymphoma and mediastinal B-cell lymphoma that shared the same mechanism [120]. In light of the fact that various small molecules that are known to inhibit various kinases are already in use as anticancer agents and these agents have been observed to decrease the histone phosphorylation, extensive research into JAK inhibitors was carried out. Ruxolitinib, which selectively inhibits JAK1 and JAK2 enzymes, 
was the first JAK inhibitor to be approved for treatment of myelofibrosis in 2011 by US-FDA. JAK1 and JAK2 are responsible for recruiting signal transducers and activators of transcription to cytokine receptors leading to modulation of gene expression. Ruxolitinib inhibits the dysregulated JAK signaling associated with myelofibrosis. The phase 3 Controlled Myelofibrosis Study with Oral JAK Inhibitor-1 (COMFORT1) and COMFORT-2 trials showed significant benefits by reducing spleen size, relieving debilitating symptoms, and improving overall survival. This drug has been hailed as a potentially blockbuster drug. It had gained very rapid acceptance among the US hematologists and oncologists.

3.6. Chromatin Remodelers as Anticancer Agents: Fool's Gold or Reality. Chromatin remodelers can be broadly divided into four main classes: the switching defective/sucrose nonfermenting (SWI/SNF) class, the imitation SWI (ISWI) class, nucleosome remodeling and deacetylation (NuRD)/Mi2/chromodomain helicase DNA binding (CHD) class, and finally inositol requiring 80 (INO80) class. These enzymes carry out relocation, removal, and exchanging of histones by utilising ATP as the energy source. These chromatinremodeling complexes play a central role in regulating various key biological phenomena, for instance, DNA replication and repair; apoptosis; chromosome segregation; and development and pluripotency.

However, it has now come to light that many of the chromatin-remodeling families may undergo mutation in various types of malignancies [121-123]. This has been further bolstered by the evidence that has emerged after cancer genomes were sequenced out. However, in the backdrop of these new findings, it becomes pertinent to be established beyond reproach that SWI/SNF mutations can lead to an imbalance between self-renewal and maturation, thus favouring a state of malignancy. Further, a lack of mechanistic insights into the process of oncogenesis induced by mutations in chromatin-remodeling complexes has put a spanner in the works and hampered the development of chromatin remodelers as effective anticancer agents.

\section{Future Challenges}

Despite the recent advances in the field of cancer epigenetics, the central maxim of tumorigenesis that it is primarily a process triggered and perpetuated by genetic anomalies remains largely unchallenged. However, notwithstanding the importance of genetic mutations, it has become clear that malignant metamorphosis of cells is governed to a large degree by the cellular environment. The packaging and the manner of packaging of genes hold as much significance as the genome itself in the modulation of cellular functions that are critical to determining cellular identity and also for producing malignant states. It has been proven beyond reproach now that the various tell-tale features of cancer like self-renewal, prevention of differentiation, skirting around cellular death, and apoptosis and tissue invasion are greatly influenced by epigenetic regulatory processes.

Despite these contentions, few questions still linger over the applicability of epigenetics as anticancer agents. Despite the assertion that epigenetic regulators may control only a few selective genes and hence could possibly affect the target genes selectively, mechanistic insights into how the epigenetic regulators target these genes continue to elude the researchers. Further, what are the features of these genes that attract the epigenetic regulators?

Additionally, from the evidences that have emerged, it is clear that epigenetic therapies have found more favour among hematological malignancies. Could this be due to the innately more complex nature of the solid malignancies? Could the intrinsically different cellular nature have anything to do with this? Are we using the epigenetic agents in the correct manner? Should the various epigenetic agents be combined together or are they better as solo therapeutic agents? The underlying biological reasons behind this need to be explored and understood urgently if epigenetic therapies are to stand a chance at finding application against solid malignancies.

In spite of these significant queries, the knowledge of epigenetic regulators has thrown up potential therapeutic marks in plenty. It is not just the various enzymes, but various modification readers, for instance, acetylation readers and methylation readers, have also attracted the attention of the pharmaceutical industry. High throughput drug discovery programs targeting cancer are being carried out [111, 124]. Though the path towards making epigenetic agents, an effective anticancer therapeutic option, is riddled with many setbacks and failures, the scientific data obtained from various epigenetic studies till date forms the bedrock on which the future of epigenetics in cancer can be built upon.

\section{Competing Interests}

The authors declare that there is no conflict of interests regarding the publication of this paper.

\section{Acknowledgments}

The authors acknowledge the support provided by Kasturba Medical College, Manipal, and Manipal University in the preparation of this paper.

\section{References}

[1] http://www.roadmapepigenomics.org/overview.

[2] P. A. Jones and S. B. Baylin, “The epigenomics of cancer," Cell, vol. 128, no. 4, pp. 683-692, 2007.

[3] P. A. Jones and P. W. Laird, "Cancer epigenetics comes of age," Nature Genetics, vol. 21, no. 2, pp. 163-167, 1999.

[4] A. Bird, "DNA methylation patterns and epigenetic memory," Genes and Development, vol. 16, no. 1, pp. 6-21, 2002.

[5] D. Takai and P. A. Jones, "Comprehensive analysis of CpG islands in human chromosomes 21 and 22," Proceedings of the National Academy of Sciences of the United States of America, vol. 99, no. 6, pp. 3740-3745, 2002.

[6] B. Brueckner and F. Lyko, "DNA methyltransferase inhibitors: old and new drugs for an epigenetic cancer therapy," Trends in Pharmacological Sciences, vol. 25, no. 11, pp. 551-554, 2004. 
[7] G. C. Prendergast and E. B. Ziff, "Methylation-sensitive sequence-specific DNA binding by the c-Myc basic region," Science, vol. 251, no. 4990, pp. 186-189, 1991.

[8] M. Curradi, A. Izzo, G. Badaracco, and N. Landsberger, "Molecular mechanisms of gene silencing mediated by DNA methylation," Molecular and Cellular Biology, vol. 22, no. 9, pp. 3157-3173, 2002.

[9] M. W. Łuczak and P. P. Jagodziński, "The role of DNA methylation in cancer development," Folia Histochemica et Cytobiologica, vol. 44, no. 3, pp. 143-154, 2006.

[10] M. Szyf, "Targeting DNA methylation in cancer," Bulletin du Cancer, vol. 93, no. 9, pp. 961-972, 2006.

[11] M. M. Suzuki and A. Bird, "DNA methylation landscapes: provocative insights from epigenomics," Nature Reviews Genetics, vol. 9, no. 6, pp. 465-476, 2008.

[12] P. A. Jones and S. B. Baylin, "The fundamental role of epigenetic events in cancer," Nature Reviews Genetics, vol. 3, no. 6, pp. 415428, 2002.

[13] A. Eden, F. Gaudet, A. Waghmare, and R. Jaenisch, "Chromosomal instability and tumors promoted by DNA hypomethylation," Science, vol. 300, no. 5618, p. 455, 2003.

[14] G. Howard, R. Eiges, F. Gaudet, R. Jaenisch, and A. Eden, "Activation and transposition of endogenous retroviral elements in hypomethylation induced tumors in mice," Oncogene, vol. 27, no. 3, pp. 404-408, 2008.

[15] A. S. Wilson, B. E. Power, and P. L. Molloy, "DNA hypomethylation and human diseases," Biochimica et Biophysica Acta, vol. 1775, no. 1, pp. 138-162, 2007.

[16] O. Ogawa, M. R. Eccles, J. Szeto et al., "Relaxation of insulin-like growth factor II gene imprinting implicated in Wilms' tumour," Nature, vol. 362, no. 6422, pp. 749-751, 1993.

[17] H. Cui, M. Cruz-Correa, F. M. Giardiello et al., "Loss of IGF2 imprinting: a potential marker of colorectal cancer risk," Science, vol. 299, no. 5613, pp. 1753-1755, 2003.

[18] V. Greger, E. Passarge, W. Höpping, E. Messmer, and B. Horsthemke, "Epigenetic changes may contribute to the formation and spontaneous regression of retinoblastoma," Human Genetics, vol. 83, no. 2, pp. 155-158, 1989.

[19] S. B. Baylin, "DNA methylation and gene silencing in cancer," Nature Clinical Practice Oncology, vol. 2, no. 1, pp. S4-S11, 2005.

[20] C. Long, B. Yin, Q. Lu et al., "Promoter hypermethylation of the RUNX3 gene in esophageal squamous cell carcinoma," Cancer Investigation, vol. 25, no. 8, pp. 685-690, 2007.

[21] Y. Akiyama, N. Watkins, H. Suzuki et al., "GATA-4 and GATA-5 transcription factor genes and potential downstream antitumor target genes are epigenetically silenced in colorectal and gastric cancer," Molecular and Cellular Biology, vol. 23, no. 23, pp. 84298439, 2003.

[22] L. Di Croce, V. A. Raker, M. Corsaro et al., "Methyltransferase recruitment and DNA hypermethylation of target promoters by an oncogenic transcription factor," Science, vol. 295, no. 5557, pp. 1079-1082, 2002.

[23] J. Frigola, J. Song, C. Stirzaker, R. A. Hinshelwood, M. A. Peinado, and S. J. Clark, "Epigenetic remodeling in colorectal cancer results in coordinate gene suppression across an entire chromosome band," Nature Genetics, vol. 38, no. 5, pp. 540-549, 2006.

[24] S. B. Baylin and P. A. Jones, "A decade of exploring the cancer epigenome-biological and translational implications," Nature Reviews Cancer, vol. 11, no. 10, pp. 726-734, 2011.
[25] H. Wu and Y. Zhang, "Mechanisms and functions of Tet proteinmediated 5-methylcytosine oxidation," Genes and Development, vol. 25, no. 23, pp. 2436-2452, 2011.

[26] P. Fenaux, G. J. Mufti, E. Hellstrom-Lindberg et al., "Efficacy of azacitidine compared with that of conventional care regimens in the treatment of higher-risk myelodysplastic syndromes: a randomised, open-label, phase III study,' The Lancet Oncology, vol. 10, no. 3, pp. 223-232, 2009.

[27] H. Kantarjlan, J.-P. J. Issa, C. S. Rosenfeld et al., "Decitabine improves patient outcomes in myelodysplastic syndromes: results of a phase III randomized study," Cancer, vol. 106, no. 8, pp. 1794-1803, 2006.

[28] H. I. Saba, M. Lubbert, and P. W. Wijermans, "Response rates of phase 2 and phase 3 trials of decitabine (DAC) in patients with myelodysplastic syndromes (MDS)," Blood, vol. 106, no. 706, 2005.

[29] P. Wijermans, M. Lübbert, G. Verhoef et al., "Low-dose 5Aza-2/-deoxycytidine, a DNA hypomethylating agent, for the treatment of high-risk myelodysplastic syndrome: a multicenter phase II study in elderly patients," Journal of Clinical Oncology, vol. 18, no. 5, pp. 956-962, 2000.

[30] L. R. Silverman, E. P. Demakos, B. L. Peterson et al., "Randomized controlled trial of azacitidine in patients with the myelodysplastic syndrome: a study of the Cancer and Leukemia Group B," Journal of Clinical Oncology, vol. 20, no. 10, pp. 24292440, 2002.

[31] J. A. Gollob and C. J. Sciambi, "Decitabine up-regulates S100A2 expression and synergizes with IFN $-\gamma$ to kill uveal melanoma cells," Clinical Cancer Research, vol. 13, no. 17, pp. 5219-5225, 2007.

[32] K. Appleton, H. J. Mackay, I. Judson et al., "Phase I and pharmacodynamic trial of the DNA methyltransferase inhibitor decitabine and carboplatin in solid tumors," Journal of Clinical Oncology, vol. 25, no. 29, pp. 4603-4609, 2007.

[33] V. Valdespino and P. M. Valdespino, "Potential of epigenetic therapies in the management of solid tumors," Cancer Management and Research, vol. 7, pp. 241-251, 2015.

[34] D. Matei, F. Fang, C. Shen et al., "Epigenetic resensitization to platinum in ovarian cancer," Cancer Research, vol. 72, no. 9, pp. 2197-2205, 2012.

[35] T. Kanda, M. Tada, F. Imazeki, O. Yokosuka, K. Nagao, and H. Saisho, "5-aza-2'-deoxycytidine sensitizes hepatoma and pancreatic cancer cell lines," Oncology Reports, vol. 14, no. 4, pp. 975-979, 2005

[36] S. Morita, S. Iida, K. Kato, Y. Takagi, H. Uetake, and K. Sugihara, "The synergistic effect of 5-aza-2'-deoxycytidine and 5-fluorouracil on drug-resistant tumors," Oncology, vol. 71, no. 5-6, pp. 437-445, 2007.

[37] K. Schmelz, M. Wagner, B. Dörken, and I. Tamm, "5-Aza-2' deoxycytidine induces p21WAF expression by demethylation of p73 leading to p53-independent apoptosis in myeloid leukemia," International Journal of Cancer, vol. 114, no. 5, pp. 683-695, 2005.

[38] X. Tang, W. Wu, S.-Y. Sun, I. I. Wistuba, W. K. Hong, and L. Mao, "Hypermethylation of the death-associated protein kinase promoter attenuates the sensitivity to TRAIL-induced apoptosis in human non-small cell lung cancer cells," Molecular Cancer Research, vol. 2, no. 12, pp. 685-691, 2004.

[39] T. J. Walton, G. Li, R. Seth, S. E. McArdle, M. C. Bishop, and R. C. Rees, "DNA demethylation and histone deacetylation inhibition co-operate to re-express estrogen receptor beta and 
induce apoptosis in prostate cancer cell-lines," Prostate, vol. 68, no. 2, pp. 210-222, 2008.

[40] A. J. Bannister and T. Kouzarides, "The CBP co-activator is a histone acetyltransferase," Nature, vol. 384, no. 6610, pp. 641643, 1996.

[41] J. Wang, H. Iwasaki, A. Krivtsov et al., "Conditional MLL-CBP targets GMP and models therapy-related myeloproliferative disease," The EMBO Journal, vol. 24, no. 2, pp. 368-381, 2005.

[42] B. J. P. Huntly, H. Shigematsu, K. Deguchi et al., "MOZ-TIF2, but not BCR-ABL, confers properties of leukemic stem cells to committed murine hematopoietic progenitors," Cancer Cell, vol. 6, no. 6, pp. 587-596, 2004.

[43] N. G. Iyer, H. Özdag, and C. Caldas, "p300/CBP and cancer," Oncogene, vol. 23, no. 24, pp. 4225-4231, 2004.

[44] L. Pasqualucci, D. Dominguez-Sola, A. Chiarenza et al., "Inactivating mutations of acetyltransferase genes in B-cell lymphoma," Nature, vol. 471, no. 7337, pp. 189-196, 2011.

[45] K. Deguchi, P. M. Ayton, M. Carapeti et al., "MOZ-TIF2induced acute myeloid leukemia requires the $\mathrm{MOZ}$ nucleosome binding motif and TIF2-mediated recruitment of CBP," Cancer Cell, vol. 3, no. 3, pp. 259-271, 2003.

[46] M. F. Fraga, E. Ballestar, A. Villar-Garea et al., "Loss of acetylation at Lys16 and trimethylation at Lys20 of histone $\mathrm{H} 4$ is a common hallmark of human cancer," Nature Genetics, vol. 37, no. 4, pp. 391-400, 2005.

[47] D. B. Seligson, S. Horvath, T. Shi et al., "Global histone modification patterns predict risk of prostate cancer recurrence," Nature, vol. 435, no. 7046, pp. 1262-1266, 2005.

[48] C. Choudhary, C. Kumar, F. Gnad et al., "Lysine acetylation targets protein complexes and co-regulates major cellular functions," Science, vol. 325, no. 5942, pp. 834-840, 2009.

[49] P. A. Cole, "Chemical probes for histone-modifying enzymes," Nature Chemical Biology, vol. 4, no. 10, pp. 590-597, 2008.

[50] R. W. Johnstone and J. D. Licht, "Histone deacetylase inhibitors in cancer therapy: is transcription the primary target?" Cancer Cell, vol. 4, no. 1, pp. 13-18, 2003.

[51] L. Bagella and M. Federico, "Histone deacetylase inhibitors in the treatment of hematological malignancies and solid tumors," Journal of Biomedicine and Biotechnology, vol. 2011, Article ID 475641, 12 pages, 2011.

[52] G. Garcia-Manero, H. M. Kantarjian, B. Sanchez-Gonzalez et al., "Phase $1 / 2$ study of the combination of 5 -aza-2' deoxycytidine with valproic acid in patients with leukemia," Blood, vol. 108, no. 10, pp. 3271-3279, 2006.

[53] S. D. Gore, S. Baylin, E. Sugar et al., "Combined DNA methyltransferase and histone deacetylase inhibition in the treatment of myeloid neoplasms," Cancer Research, vol. 66, no. 12, pp. 6361-6369, 2006.

[54] S. D. Gore, L.-J. Weng, S. Zhai et al., "Impact of the putative differentiating agent sodium phenylbutyrate on myelodysplastic syndromes and acute myeloid leukemia," Clinical Cancer Research, vol. 7, no. 8, pp. 2330-2339, 2001.

[55] A. Kuendgen, C. Strupp, M. Aivado et al., "Treatment of myelodysplastic syndromes with valproic acid alone or in combination with all-trans retinoic acid," Blood, vol. 104, no. 5, pp. 1266-1269, 2004

[56] M. Bishton, M. Kenealy, R. Johnstone, W. Rasheed, and H. M. Prince, "Epigenetic targets in hematological malignancies: combination therapies with HDACis and demethylating agents," Expert Review of Anticancer Therapy, vol. 7, no. 10, pp. 14391449, 2007.
[57] W. K. Kelly, V. M. Richon, O. O’Connor et al., "Phase I clinical trial of histone deacetylase inhibitor: suberoylanilide hydroxamic acid administered intravenously," Clinical Cancer Research, vol. 9, no. 10, pp. 3578-3588, 2003.

[58] M. Duvic, R. Talpur, X. Ni et al., "Phase 2 trial of oral vorinostat (suberoylanilide hydroxamic acid, SAHA) for refractory cutaneous T-cell lymphoma (CTCL)," Blood, vol. 109, no. 1, pp. 3139, 2007.

[59] E. A. Olsen, Y. H. Kim, T. M. Kuzel et al., "Phase IIB multicenter trial of vorinostat in patients with persistent, progressive, or treatment refractory cutaneous T-cell lymphoma," Journal of Clinical Oncology, vol. 25, no. 21, pp. 3109-3115, 2007.

[60] C. L. Andersen, M. F. Mcmullin, E. Ejerblad et al., "A phase II study of vorinostat (MK-0683) in patients with polycythaemia vera and essential thrombocythaemia," British Journal of Haematology, vol. 162, no. 4, pp. 498-508, 2013.

[61] H. Akada, S. Akada, A. Gajra et al., "Efficacy of vorinostat in a murine model of polycythemia vera," Blood, vol. 119, no. 16, pp. 3779-3789, 2012.

[62] G. Garcia-Manero, F. P. Tambaro, N. B. Bekele et al., "Phase II trial of vorinostat with idarubicin and cytarabine for patients with newly diagnosed acute myelogenous leukemia or myelodysplastic syndrome," Journal of Clinical Oncology, vol. 30, no. 18, pp. 2204-2210, 2012.

[63] M. Ogura, K. Ando, T. Suzuki et al., "A multicentre phase II study of vorinostat in patients with relapsed or refractory indolent B-cell non-Hodgkin lymphoma and mantle cell lymphoma," British Journal of Haematology, vol. 165, no. 6, pp. 768776, 2014.

[64] D. S. Siegel, P. Richardson, M. Dimopoulos et al., "Vorinostat in combination with lenalidomide and dexamethasone in patients with relapsed or refractory multiple myeloma," Blood Cancer Journal, vol. 4, article e182, 2014.

[65] P. N. Munster, K. T. Thurn, S. Thomas et al., "A phase II study of the histone deacetylase inhibitor vorinostat combined with tamoxifen for the treatment of patients with hormone therapyresistant breast cancer," British Journal of Cancer, vol. 104, no. 12, pp. 1828-1835, 2011.

[66] R. Furumai, A. Matsuyama, N. Kobashi et al., "FK228 (depsipeptide) as a natural prodrug that inhibits class I histone deacetylases," Cancer Research, vol. 62, no. 17, pp. 4916-4921, 2002.

[67] J. C. Byrd, G. Marcucci, M. R. Parthun et al., "A phase 1 and pharmacodynamic study of depsipeptide (FK228) in chronic lymphocytic leukemia and acute myeloid leukemia," Blood, vol. 105, no. 3, pp. 959-967, 2005.

[68] R. L. Piekarz, R. Robey, V. Sandor et al., "Inhibitor of histone deacetylation, depsipeptide (FR901228), in the treatment of peripheral and cutaneous T-cell lymphoma: a case report," Blood, vol. 98, no. 9, pp. 2865-2868, 2001.

[69] R. L. Piekarz, R. Frye, M. Turner et al., "Phase II multiinstitutional trial of the histone deacetylase inhibitor romidepsin as monotherapy for patients with cutaneous T-cell lymphoma," Journal of Clinical Oncology, vol. 27, no. 32, pp. 5410-5417, 2009.

[70] S. J. Whittaker, M.-F. Demierre, E. J. Kim et al., "Final results from a multicenter, international, pivotal study of romidepsin in refractory cutaneous T-cell lymphoma," Journal of Clinical Oncology, vol. 28, no. 29, pp. 4485-4491, 2010.

[71] R. L. Piekarz, A. R. Frye, J. J. Wright et al., "Cardiac studies in patients treated with depsipeptide, FK228, in a phase II trial for 
T-cell lymphoma," Clinical Cancer Research, vol. 12, no. 12, pp. 3762-3773, 2006.

[72] G. Garcia-Manero, S. Assouline, J. Cortes et al., "Phase 1 study of the oral isotype specific histone deacetylase inhibitor MGCD0103 in leukemia," Blood, vol. 112, no. 4, pp. 981-989, 2008.

[73] J. E. Lancet, G. Nichols, S. Assouline et al., "A phase I study of MGCD0103 given as a twice weekly oral dose in patients with advanced leukemias or myelodysplastic syndromes (MDS)," Journal of Clinical Oncology, vol. 25, no. 18, supplement 2516, 2007.

[74] R. G. Bociek, J. Kuruvilla, B. Pro et al., "Isotype-selective histone deacetylase (HDAC) inhibitor MGCD0103 demonstrates clinical activity and safety in patients with relapsed/refractory classical Hodgkin Lymphoma (HL)," Journal of Clinical Oncology, vol. 26, supplement 8507, no. 15, 2008.

[75] X. Li, J. Zhang, Y. Xie, Y. Jiang, Z. Yingjie, and W. Xu, "Progress of HDAC inhibitor panobinostat in the treatment of cancer," Current Drug Targets, vol. 15, no. 6, pp. 622-634, 2014.

[76] L. V. Rhodes, C. R. Tate, H. C. Segar et al., "Suppression of triple-negative breast cancer metastasis by pan-DAC inhibitor panobinostat via inhibition of ZEB family of EMT master regulators," Breast Cancer Research and Treatment, vol. 145, no. 3, pp. 593-604, 2014.

[77] P. Tan, A. Wei, S. Mithraprabhu et al., "Dual epigenetic targeting with panobinostat and azacitidine in acute myeloid leukemia and high-risk myelodysplastic syndrome," Blood Cancer Journal, vol. 4, article e170, 2014.

[78] M. Duvic, R. Dummer, J. C. Becker et al., "Panobinostat activity in both bexarotene-exposed and -naive patients with refractory cutaneous T-cell lymphoma: results of a phase II trial," European Journal of Cancer, vol. 49, no. 2, pp. 386-394, 2013.

[79] L. Ellis, Y. Pan, G. K. Smyth et al., "Histone deacetylase inhibitor panobinostat induces clinical responses with associated alterations in gene expression profiles in cutaneous T-cell lymphoma," Clinical Cancer Research, vol. 14, no. 14, pp. 45004510, 2008.

[80] A. Younes, A. Sureda, D. Ben-Yehuda et al., "Panobinostat in patients with relapsed/refractory Hodgkin's lymphoma after autologous stem-cell transplantation: results of a phase II study," Journal of Clinical Oncology, vol. 30, no. 18, pp. 2197-2203, 2012.

[81] I. M. Ghobrial, F. Campigotto, T. J. Murphy et al., "Results of a phase 2 trial of the single-agent histone deacetylase inhibitor panobinostat in patients with relapsed/refractory Waldenström macroglobulinemia," Blood, vol. 121, no. 8, pp. 1296-1303, 2013.

[82] V. T. Hungria, S. S. Yoon, M. Beksac et al., "Panobinostat plus bortezomib and dexamethasone versus placebo plus bortezomib and dexamethasone in patients with relapsed or relapsed and refractory multiple myeloma: a multicentre, randomised, double-blind phase 3 trial," The Lancet Oncology, vol. 15, no. 11, pp. 1195-1206, 2014.

[83] P. G. Richardson, R. L. Schlossman, M. Alsina et al., "PANORAMA 2: panobinostat in combination with bortezomib and dexamethasone in patients with relapsed and bortezomib-refractory myeloma," Blood, vol. 122, no. 14, pp. 23312337, 2013.

[84] H. J. Mackay, H. Hirte, T. Colgan et al., "Phase II trial of the histone deacetylase inhibitor belinostat in women with platinum resistant epithelial ovarian cancer and micropapillary (LMP) ovarian tumours," European Journal of Cancer, vol. 46, no. 9, pp. 1573-1579, 2010.
[85] A. Cashen, M. Juckett, A. Jumonville et al., "Phase II study of the histone deacetylase inhibitor belinostat (PXD101) for the treatment of myelodysplastic syndrome (MDS)," Annals of hematology, vol. 91, no. 1, pp. 33-38, 2012.

[86] M. Takaoka, "Of the phenolic substances of white hellebore (Veratrum grandiflorum Loes. Fil.)," Journal of the Faculty of Science, Hokkaido Imperial University, vol. 3, pp. 1-16, 1940.

[87] K. B. Harikumar, A. B. Kunnumakkara, G. Sethi et al., "Resveratrol, a multitargeted agent, can enhance antitumor activity of gemcitabine in vitro and in orthotopic mouse model of human pancreatic cancer," International Journal of Cancer, vol. 127, no. 2, pp. 257-268, 2010.

[88] M. Fukui, N. Yamabe, and B. T. Zhu, "Resveratrol attenuates the anticancer efficacy of paclitaxel in human breast cancer cells in vitro and in vivo," European Journal of Cancer, vol. 46, no. 10, pp. 1882-1891, 2010.

[89] T. Kubota, Y. Uemura, M. Kobayashi, and H. Taguchi, "Combined effects of resveratrol and paclitaxel on lung cancer cells," Anticancer Research, vol. 23, no. 5, pp. 4039-4046, 2003.

[90] S. H. Kweon, J. H. Song, and T. S. Kim, "Resveratrol-mediated reversal of doxorubicin resistance in acute myeloid leukemia cells via downregulation of MRP1 expression," Biochemical and Biophysical Research Communications, vol. 395, no. 1, pp. 104110, 2010.

[91] S. C. Lee, J. Y. Chan, and S. Pervaiz, "Spontaneous and 5-fluorouracil-induced centrosome amplification lowers the threshold to resveratrol-evoked apoptosis in colon cancer cells," Cancer Letters, vol. 288, no. 1, pp. 36-41, 2010.

[92] S. C. Gupta, R. Kannappan, S. Reuter, J. H. Kim, and B. B. Aggarwal, "Chemosensitization of tumors by resveratrol," Annals of the New York Academy of Sciences, vol. 1215, no. 1, pp. 150-160, 2011.

[93] O. R. Bereshchenko, W. Gu, and R. Dalla-Favera, "Acetylation inactivates the transcriptional repressor BCL6," Nature Genetics, vol. 32, no. 4, pp. 606-613, 2002.

[94] C.-W. Chung and J. Witherington, "Progress in the discovery of small-molecule inhibitors of bromodomain-histone interactions," Journal of Biomolecular Screening, vol. 16, no. 10, pp. 11701185, 2011.

[95] M. A. Dawson and T. Kouzarides, "Cancer epigenetics: from mechanism to therapy," Cell, vol. 150, no. 1, pp. 12-27, 2012.

[96] P. Filippakopoulos, J. Qi, S. Picaud et al., "Selective inhibition of BET bromodomains," Nature, vol. 468, no. 7327, pp. 1067-1073, 2010.

[97] J. A. Mertz, A. R. Conery, B. M. Bryant et al., "Targeting MYC dependence in cancer by inhibiting BET bromodomains," Proceedings of the National Academy of Sciences of the United States of America, vol. 108, no. 40, pp. 16669-16674, 2011.

[98] M. A. Dawson, R. K. Prinjha, A. Dittmann et al., "Inhibition of BET recruitment to chromatin as an effective treatment for MLL-fusion leukaemia," Nature, vol. 478, no. 7370, pp. 529-533, 2011.

[99] J. Zuber, J. Shi, E. Wang et al., "RNAi screen identifies Brd4 as a therapeutic target in acute myeloid leukaemia," Nature, vol. 478, no. 7370, pp. 524-528, 2011.

[100] J. E. Delmore, G. C. Issa, M. E. Lemieux et al., "BET bromodomain inhibition as a therapeutic strategy to target $c$-myc," Cell, vol. 146, no. 6, pp. 904-917, 2011.

[101] N. Meyer and L. Z. Penn, "Reflecting on 25 years with MYC," Nature Reviews Cancer, vol. 8, no. 12, pp. 976-990, 2008. 
[102] A. V. Krivtsov and S. A. Armstrong, "MLL translocations, histone modifications and leukaemia stem-cell development," Nature Reviews Cancer, vol. 7, no. 11, pp. 823-833, 2007.

[103] R. Margueron and D. Reinberg, "The Polycomb complex PRC2 and its mark in life," Nature, vol. 469, no. 7330, pp. 343-349, 2011.

[104] C. J. Sneeringer, M. P. Scott, K. W. Kuntz et al., "Co-ordinated activities of wild type plus mutant EZH2 drive tumor-associated hypertrimethylation of lysine 27 on histone $\mathrm{H} 3$ (H3K27) in human B-cell lymphomas," Proceedings of the National Academy of Science of United States of America, vol. 107, no. 49, pp. $20980-$ 20985, 2010.

[105] T. Ernst, A. J. Chase, J. Score et al., "Inactivating mutations of the histone methyltransferase gene EZH2 in myeloid disorders," Nature Genetics, vol. 42, no. 8, pp. 722-726, 2010.

[106] G. Nikoloski, S. M. C. Langemeijer, R. P. Kuiper et al., "Somatic mutations of the histone methyltransferase gene EZH2 in myelodysplastic syndromes," Nature Genetics, vol. 42, no. 8, pp. 665-667, 2010.

[107] P. Ntziachristos, A. Tsirigos, P. V. Vlierberghe et al., "Genetic inactivation of the polycomb repressive complex 2 in $\mathrm{T}$ cell acute lymphoblastic leukemia," Nature Medicine, vol. 18, no. 2, pp. 296-301, 2012.

[108] J. Zhang, L. Ding, L. Holmfeldt et al., "The genetic basis of early T-cell precursor acute lymphoblastic leukemia," Nature, vol. 481, pp. 157-163, 2012.

[109] S. R. Daigle, E. J. Olhava, C. A. Therkelsen et al., "Selective killing of mixed lineage leukemia cells by a potent smallmolecule DOT1L inhibitor," Cancer Cell, vol. 20, no. 1, pp. 53-65, 2011.

[110] N. Mosammaparast and Y. Shi, "Reversal of histone methylation: biochemical and molecular mechanisms of histone demethylases," Annual Review of Biochemistry, vol. 79, pp. 155$179,2010$.

[111] J. Barretina, G. Caponigro, N. Stransky et al., “The cancer cell line encyclopedia enables predictive modelling of anticancer drug sensitivity," Nature, vol. 483, no. 7391, pp. 603-607, 2012.

[112] T. Schenk, W. C. Chen, S. Göllner et al., "Inhibition of the LSD1 (KDM1A) demethylase reactivates the all-trans-retinoic acid differentiation pathway in acute myeloid leukemia," Nature Medicine, vol. 18, no. 4, pp. 605-611, 2012.

[113] S. D. Taverna, H. Li, A. J. Ruthenburg, C. D. Allis, and D. J. Patel, "How chromatin-binding modules interpret histone modifications: lessons from professional pocket pickers," Nature Structural and Molecular Biology, vol. 14, no. 11, pp. 1025-1040, 2007.

[114] G. K. Dialynas, M. W. Vitalini, and L. L. Wallrath, "Linking Heterochromatin Protein 1 (HP1) to cancer progression," Mutation Research, vol. 647, no. 1-2, pp. 13-20, 2008.

[115] A. H. Coles and S. N. Jones, "The ING gene family in the regulation of cell growth and tumorigenesis," Journal of Cellular Physiology, vol. 218, no. 1, pp. 45-57, 2009.

[116] G. G. Wang, J. Song, Z. Wang et al., "Haematopoietic malignancies caused by dysregulation of a chromatin-binding PHD finger," Nature, vol. 459, no. 7248, pp. 847-851, 2009.

[117] S. H. Baek, "When signaling kinases meet histones and histone modifiers in the nucleus," Molecular Cell, vol. 42, no. 3, pp. 274284, 2011.

[118] D. Bungard, B. J. Fuerth, P.-Y. Zeng et al., "Signaling kinase AMPK activates stress-promoted transcription via histone $\mathrm{H} 2 \mathrm{~B}$ phosphorylation," Science, vol. 329, no. 5996, pp. 1201-1205, 2010.
[119] M. A. Dawson, A. J. Bannister, B. Göttgens et al., "JAK2 phosphorylates histone H3Y41 and excludes HP1 $\alpha$ from chromatin," Nature, vol. 461, no. 7265, pp. 819-822, 2009.

[120] L. Rui, N. C. T. Emre, M. J. Kruhlak et al., "Cooperative epigenetic modulation by cancer amplicon genes," Cancer Cell, vol. 18, no. 6, pp. 590-605, 2010.

[121] I. Versteege, N. Sévenet, J. Lange et al., “Truncating mutations of hSNF5/INI1 in aggressive paediatric cancer," Nature, vol. 394, no. 6689, pp. 203-206, 1998.

[122] B. G. Wilson and C. W. M. Roberts, "SWI/SNF nucleosome remodellers and cancer," Nature Reviews Cancer, vol. 11, no. 7, pp. 481-492, 2011.

[123] D.-Q. Li, S. B. Pakala, S. S. Nair, J. Eswaran, and R. Kumar, "Metastasis-associated protein 1/nucleosome remodeling and histone deacetylase complex in cancer," Cancer Research, vol. 72, no. 2, pp. 387-394, 2012.

[124] M. J. Garnett, E. J. Edelman, S. J. Heidorn et al., "Systematic identification of genomic markers of drug sensitivity in cancer cells," Nature, vol. 483, no. 7391, pp. 570-575, 2012. 

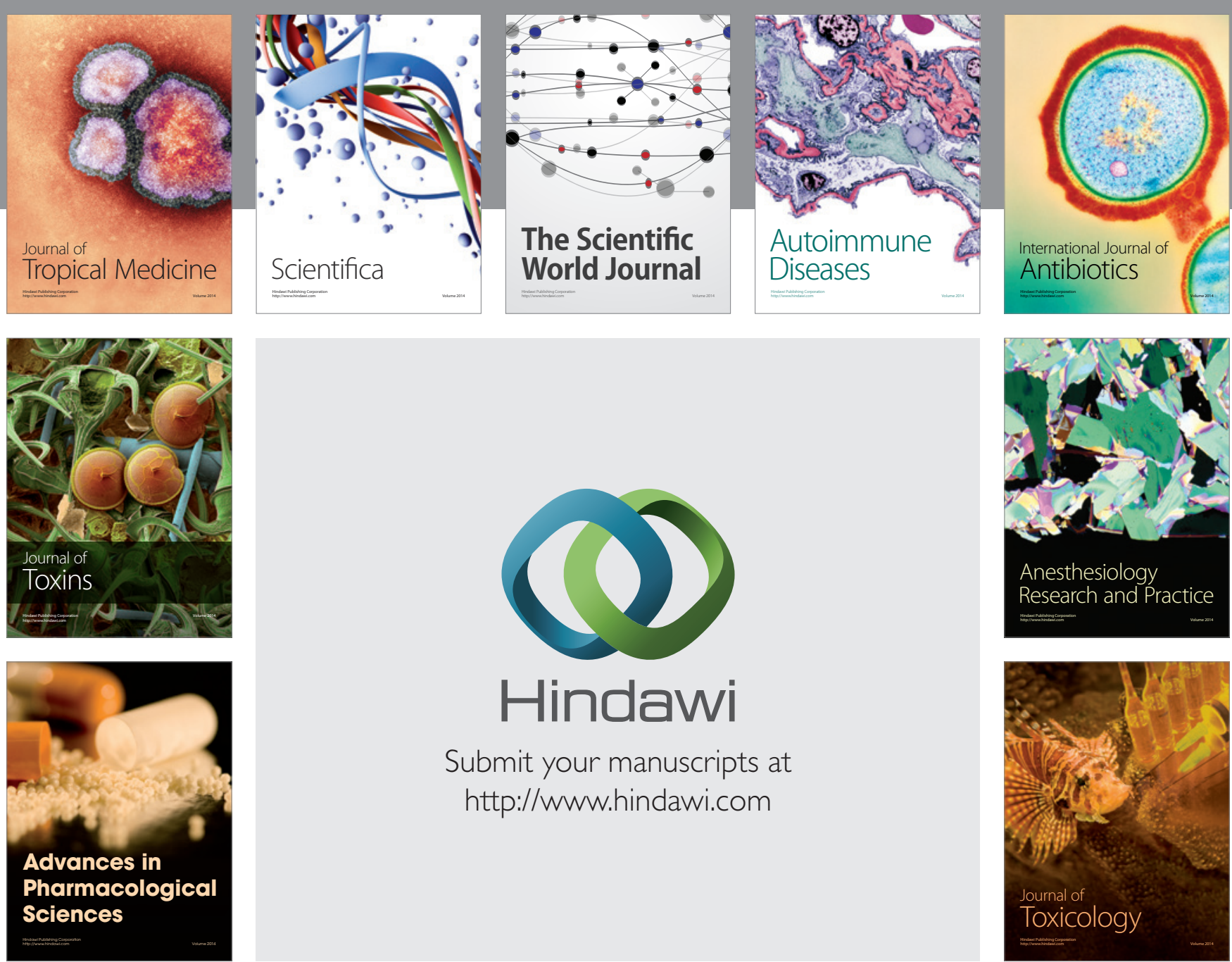

\section{Hindawi}

Submit your manuscripts at

http://www.hindawi.com
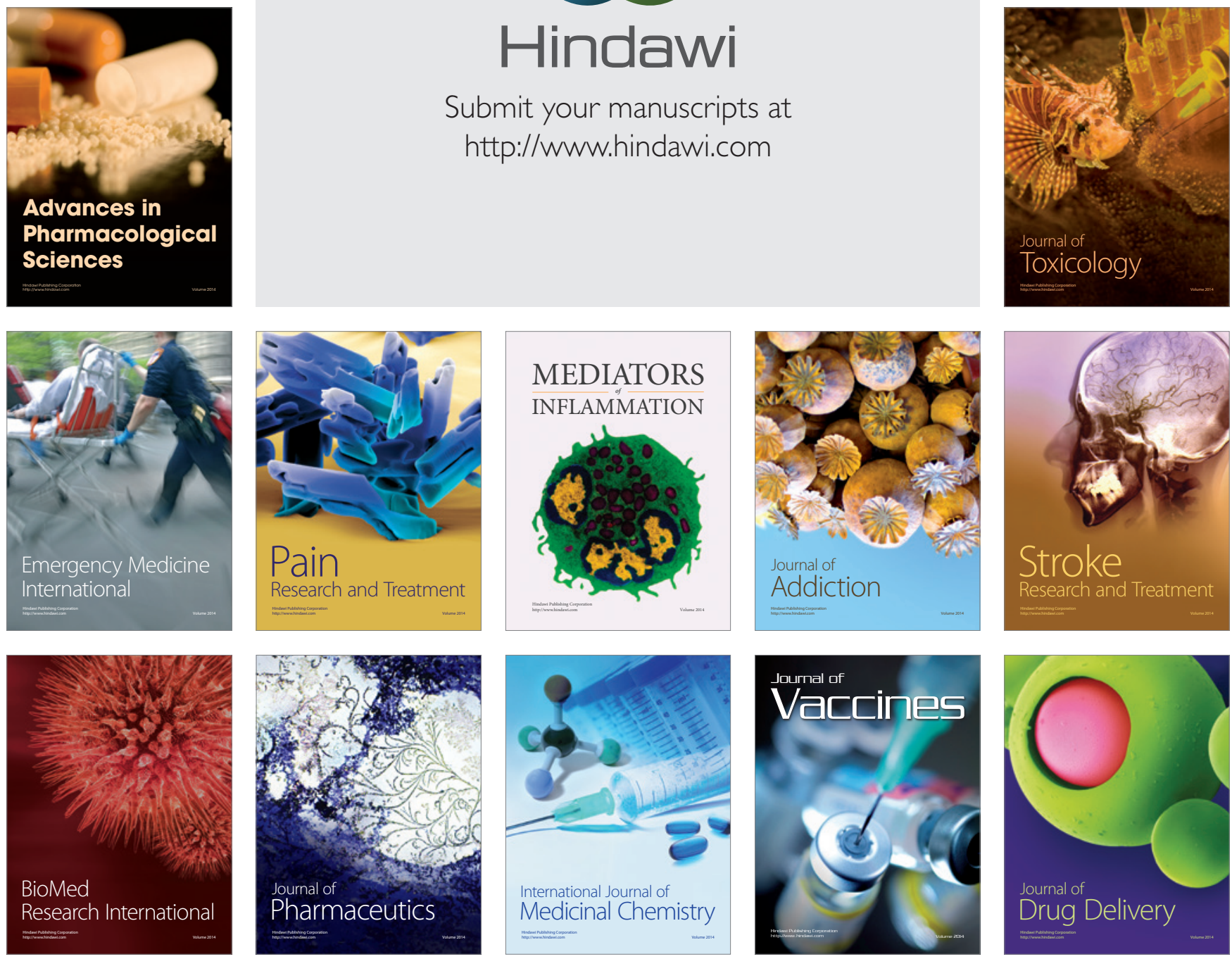